\title{
Fetal Alcohol Spectrum Disorder (FASD): A call on mental health treatment professionals to become informed
}

\author{
Jerrod Brown ${ }^{1,2,3 *}$, Diane Harr ${ }^{1}$, Stephen Morgan ${ }^{1}$, Stefanie Varga ${ }^{4}$ and Amanda Fenrich ${ }^{5}$ \\ ${ }^{1}$ Concordia University, St. Paul, MN, USA \\ ${ }^{2}$ Pathways Counseling Center, St. Paul, MN, USA \\ ${ }^{3}$ The American Institute for the Advancement of Forensic Studies, St. Paul, MN, USA \\ ${ }^{4}$ Treehouse Psychology, Hugo, MN, USA \\ ${ }^{5}$ Washington State Department of Corrections, Monroe, WA, USA
}

\begin{abstract}
Fetal Alcohol Spectrum Disorder (FASD) is a spectrum of life-long disorders caused by prenatal alcohol exposure. The adverse effects of FASD include cognitive, behavioral, emotional, physical, and adaptive functioning deficits. Since the deficits and symptoms of FASD can have a deleterious impact on the individual's overall daily functioning over the lifespan, assistance from a qualified mental health professional is often required. While FASD can be potentially debilitating, long-term positive outcomes have been associated with early intervention and diagnosis provided by adequately trained mental health professionals. This article provides the reader with an introductory overview of FASD and its implications for mental health treatment professionals, training recommendations, and suggestions for future research.
\end{abstract}

\section{Fetal Alcohol Spectrum Disorder (FASD)}

Afflicting millions of individuals in the United States and many more around the world, Fetal Alcohol Spectrum Disorder (FASD) is a life-course persistent disorder caused by prenatal alcohol exposure [1-4]. It has been estimated that disorders associated with prenatal alcohol exposure impact up to $5 \%$ of the general population [5] with even higher rates being documented among at-risk and criminal justice populations [6-9]. Alcohol consumed when a woman is pregnant is passed to the developing fetus that can result in significant physical and neurological effects. For this reason, it is never safe to drink alcohol when trying to get pregnant or during pregnancy. FASD can include a wide range of cognitive (e.g., intelligence, executive functions, shortand long-term memory, and attention), social (e.g., communication skills and gullibility), physical (e.g., reduced height and weight, and smaller head size), and adaptive impairments (e.g., decision making and problem-solving abilities) [10-14]. Of critical concern is the disruption of immune system development that leads to increased risk of disease and infection throughout the exposed individual's life [15], with deleterious effects of FASD presenting not only in the individual, but also on the family and in the community. These effects include negative school experiences, legal problems and involvement in the criminal system, inappropriate sexual behavior, and drug and alcohol problems [16].

No universal set of FASD criteria fits all cases given that the appearance and severity of symptoms varies as a function of the prenatal exposure to alcohol. In addition to these symptoms, individuals with FASD often have comorbid behavioral (e.g., ADHD and conduct disorder), mood (i.e., major depression and bipolar disorder), anxiety, and substance use disorders $[12,17,18]$. This complex interplay of comorbid psychiatric symptoms makes screening and assessment increasingly challenging, which in turn can increase the likelihood of missed diagnosis or misdiagnosis leading to negative long-term outcomes. In light of these pervasive difficulties, FASD is likely prevalent in inpatient and outpatient mental health and substance use treatment facilities. To help combat these issues, the Diagnostic and Statistical Manual-5th Edition [19] recently introduced Neurodevelopmental Disorder Associated with Prenatal Alcohol Exposure (ND-PAE) as a disorder for future study.

\section{Implications for mental health professionals}

The presence of FASD may not be immediately clear during initial encounters with a client. To combat this obfuscation, there are several steps that require consideration by a mental health professional $[20,21]$. First, mental health professionals should not rely exclusively on intelligence measures in their assessment batteries. Intelligence tests may not accurately detect the adaptive functioning deficits of FASD. Second, the manner by which a mental health professional interviews a client is important. For example, FASD-related deficits are often masked by an over-reliance on simple yes/no questions. Third, a clinical professional should be mindful of the impact of FASD symptoms on assessment strategies [22]. One consideration, is memory-related impairments (i.e., memory loss, suggestibility, and confabulation) and the association with reported misinformation

Correspondence to: Jerrod Brown, Ph.D., MA, MS, MS, MS, Pathways Counseling Center, 1919 University Ave. W. Suite 6 St. Paul MN, 55104, USA, E-mail: jerrod01234brown@live.com

Key words: fetal alcohol spectrum disorder, mental health professional, ND-PAE, research, training

Received: December 04, 2017; Accepted: December 27, 2017; Published: December 30, 2017 
that muddles the evaluation process [23,24]. Finally, the assessment of FASD often necessitates nuanced differential diagnosis, which may be beyond the skill sets of some professionals and thus requires consultation with an FASD expert. The implications for failing to account for these diagnostic complications results in misdiagnosis of FASD. In turn, effected individuals may receive inappropriate treatment with a decrease in the likelihood of treatment success and positive outcomes [25].

To maximize the possibility of treatment success, treatment plans should be individualized in a developmentally appropriate manner that considers the symptoms and difficulties of FASD. Such plans should avoid the incorporation of insight-based therapeutic and group therapy approaches. In contrast, clients with FASD may benefit from the utilization of attachment-based approaches that emphasizes the development of life-skills, self-advocacy, social skills, sleep improvement, and behavioral regulation. A trauma-informed approach may be particularly fruitful given that the symptoms of FASD may predispose clients to higher rates of victimization and trauma than the general population [26,27]. Given the range of expertise such a treatment endeavor necessitates, mental health professionals may need to develop and coordinate a network of professionals that include those who work in health care, assisted living, financial management, psychotherapy, and case management.

\section{Training recommendations}

There are a number of myths, misconceptions, and incorrect information regarding FASD within the general population. It is important to recognize that this disorder is one that results in permanent brain impairment with associated disabilities. The primary goal of any FASD-related training is to familiarize mental health professionals with accurate and truthful information regarding FASD. This includes understanding the red flags and risk factors associated with the disorder, symptoms, and consequences. Mental health professionals should receive training in several key areas: understanding the need for early diagnosis and intervention, awareness regarding the most up to date empirical research findings, exploration of both standard and newly developed screening tools and measures, and established best practices for treating individuals with FASD. Mental health professionals should also be educated about the Diagnostic and Statistical Manual of Mental Disorders fifth edition (DSM-5) definition of Neurobehavioral Disorder associated with Prenatal Alcohol Exposure (ND-PAE) criteria. This designation serves as a diagnostic criteria and can be used in cases of suspected prenatal alcohol exposure. Training should educate mental health professionals regarding the various secondary conditions and co-occurring disorders often experienced by individuals impacted by FASD. In particular, understanding of effective intervention approaches and best-practice (e.g., executive and adaptive functioning, attachmentbased interventions and trauma-informed care approaches) treatment options for individuals impacted by FASD who are receiving services in a mental health treatment setting is imperative. It is also critical that mental health professionals understand how individuals impacted by FASD may experience significant disadvantages in treatment when providers lack appropriate awareness and understanding of the disorder. Individuals impacted by FASD can and do respond to treatment, so every effort should be made to develop a plan that is comprehensive and holistic in nature. Finally, training should conclude with a discussion of existing gaps in knowledge and the identification of directions for future research. Advanced training in these areas should assist mental health professionals in the identification and treatment of individuals impacted by prenatal alcohol exposure.

\section{Suggestions for further research}

The avenues for additional research into FASD are nearly limitless. One area most in need of research includes the specific deficits and symptoms that most impact mental health treatment outcomes. Also, of value to researchers are the perceptions and experiences of various stakeholders involved in the individual's life. Learning about the perceptions and experiences of FASD among criminal justice, mental health, and other related field professionals is necessary in advancing the identification and treatment of individuals impacted by FASD. Similarly, a survey of patients' perceptions and experiences as participants in various inpatient and outpatient treatment programs may provide additional insights. The perceptions and experiences of the various family members can also be helpful. While not having FASD themselves, the family often sees the impact of what works, and conversely what does not work with their family member in terms of treatment and medication. Training is another area of FASD research in need of further investigation. What are the training needs of the professionals in the field? Are their training needs being met? What would help professionals meet their clients with FASD needs to ensure better outcomes? What are the specific needs related to managing individuals with FASD?

Prevalence and incidence rates of FASD are also tremendously valuable pieces of information. Accurately determining both prevalence and incidence rates of FASD across various mental health settings, populations, and environments would be very helpful to the field at large. This would include additional studies examining FASD prevalence rates in inpatient, outpatient and substance use treatment programs, correctional settings, detoxication centers, homeless shelters, probation and parole populations, and domestic violence advocacy centers. Lastly, in focusing on mental health professionals, an investigation on the efficacy of incorporating and using the ND-PAE criteria in identifying and treating patients with suspected or confirmed FASD may be critically relevant. This would be especially valuable with respect to developing treatment plans for clients with FASD. Research such as this could lead to the development of a FASD-based screening tool for use specifically by mental health treatment providers.

\section{Conclusion}

Awareness of Fetal Alcohol Spectrum Disorder (FASD) is rising among mental health professionals. With the inclusion of ND-PAE in the DSM-5 practitioners now have a diagnostic criteria for individuals presenting with an FASD-related disorder. These diagnostic criteria cover a wide range of symptoms including cognitive, social, and adaptive impairments. While not always initially apparent, clinicians can employ a number of tests and assessments to help aid in diagnosis. Each client with FASD will be unique and the degree of brain damage can vary. The treatment plan and management of the client must be tailored to the patient for maximum efficacy. In order to provide the best service to each client with FASD, it is important that mental health professionals be adequately trained about FASD. If the mental health professional is not well-trained, the cost to the client who is misdiagnosed could be immeasurable. Therefore, it is essential that stakeholders and those working with this population are current and informed on the most effective treatment methods. Appropriate training of mental health professionals is likely to result in potential further research regarding FASD. The study of the perceptions and experiences of various FASD stakeholders may also provide numerous insights. Lastly, establishing the utility and value of the ND-PAE criteria among chemical and mental health professionals is likely to greatly enhance its acceptance within the mental health community. 


\section{References}

1. Charness ME, Riley EP, Sowell ER(2016) Drinking During Pregnancy and the Developing Brain: Is Any Amount Safe? Trends Cogn Sci 20: 80-82. [Crossref]

2. May PA, Baete A, Russo J, Elliott AJ, Blankenship J, et al. (2014) Prevalence and characteristics of Fetal Alcohol Spectrum Disorders. Pediatrics 134: 855-866. [Crossref]

3. May PA, De Vries MM, Marais AS, Kalberg WO, Buckley D, et al. (2017) Replication of High Fetal Alcohol Spectrum Disorders Prevalence Rates, Child Characteristics, and Maternal Risk Factors in a Second Sample of Rural Communities in South Africa. Int J Environ Res Public Health 14: 522. [Crossref]

4. Petrenko CL, Tahir N, Mahoney EC, Chin NP (2014) Prevention of secondary conditions in Fetal Alcohol Spectrum Disorders: Identification of systems-level barriers. Matern Child Health J 18: 1496-1505. [Crossref]

5. May PA, Gossage JP, Kalberg WO, Robinson LK, Buckley D, et al. (2009) Prevalence and epidemiologic characteristics of FASD from various research methods with an emphasis on recent in-school studies. Dev Disabil Res Rev 15: 176-192. [Crossref]

6. Brown J, Cich J, Singh J (2017) Perceptions of FASD by Minnesota public defenders. Adv Ment Health Intellect Disabil 11: 165-172.

7. Burd L, Fast D, Conry J (2010) Fetal alcohol spectrum disorder as a marker for increased risk of involvement with correction systems. J Psychiatry Law 38: 559-584.

8. Fast DK, Conry J, Loock CA (1999) Identifying fetal alcohol syndrome among youth in the criminal justice system. J Dev Behav Pediatr 20: 370-372. [Crossref]

9. Rangmar J, Sandberg AD, Aronson M, Fahlke C (2017) Self-reported health, use of alcohol and illicit drugs, and criminality among adults with foetal alcohol syndrome. Nordic Studies on Alcohol and Drugs, 1455072517707887.

10. Fagerlund Å, Autti-Rämö I, Kalland M, Santtila P, Hoyme HE, et al. (2012) Adaptive behaviour in children and adolescents with foetal alcohol spectrum disorders: a comparison with specific learning disability and typical development. Eur Child Adolesc Psychiatry 21: 221-231. [Crossref]

11. Chasnoff IJ, Wells AM, King L (2015) Misdiagnosis and missed diagnoses in foster and adopted children with prenatal alcohol exposure. Pediatrics 135: 264-270.

12. Lange S, Rovet J, Rehm J, Popova S (2017) Neurodevelopmental profile of fetal alcohol spectrum disorder: A systematic review. BMC Psychol 5: 1-12. [Crossref]

13. Mattson SN, Roesch SC, Glass L, Deweese BN, Coles CD, et al. (2013) Further development of a neurobehavioral profile of fetal alcohol spectrum disorders. Alcohol Clin Exp Res 37: 517-528. [Crossref]
14. Price A, Cook PA, Norgate S, Mukherjee R (2017) Prenatal alcohol exposure and traumatic childhood experiences: A systematic review. Neurosci Biobehav Rev 80: 8998. [Crossref]

15. Theresa W. Gauthier (2015) Prenatal alcohol Exposure and the Developing Immune System. Alcohol Res 37: 279- 286. [Crossref]

16. Streissguth AP, Bookstein FL, Barr HM, Sampson PD, O’Malley K, et al. (2004) Risk factors for adverse life outcomes in fetal alcohol syndrome and fetal life effects. J Dev Behav Pediatr 25: 228-238. [Crossref]

17. Famy C, Streissguth AP, Unis AS (1998) Mental illness in adults with fetal alcohol syndrome or fetal alcohol effects. Am J Psychiatry 155: 552-554. [Crossref]

18. Rasmussen C, Benz J, Pei J, Andrew G, Schuller G, et al. (2010) The impact of an ADHD co-morbidity on the diagnosis of FASD. Can J Clin Pharmacol 17: 165-176. [Crossref]

19. Kable JA, Mukherjee RA (2017) Neurodevelopmental disorder associated with prenatal exposure to alcohol (ND-PAE): A proposed diagnostic method of capturing the neurocognitive phenotype of FASD. Eur J Med Genet 60: 49-54. [Crossref]

20. Clarke ME, Gibbard WB (2003) Overview of fetal alcohol spectrum disorders for mental health professionals. Can Child Adolesc Psychiatr Rev 12: 57-63. [Crossref]

21. Lockhart PJ (2001) Fetal alcohol spectrum disorders for mental health professionals-a brief review. Curr Opin Psychiatry 14: 463-469.

22. Brown JM, Haun J, Zapf PA, Brown NN (2017) Fetal Alcohol Spectrum Disorders (FASD) and competency to stand trial (CST): Suggestions for a 'best practices' approach to forensic evaluation. Int J Law Psychiatry 52: 19-27. [Crossref]

23. Brown J (2017) Fetal Alcohol Spectrum Disorder and Confabulation: A Clinical, Forensic, and Judicial Dilemma. The Journal of Special Populations 1: 1-11.

24. Brown N, Gudjonsson G, Connor P (2011) Suggestibility and fetal alcohol spectrum disorders: i'll tell you anything you want to hear. J Psychiatry Law 1: 39-73.

25. Petrenko CL, Alto ME (2017) Interventions in fetal alcohol spectrum disorders: An international perspective. Eur J Med Genet 60: 79-91. [Crossref]

26. Henry J, Sloane M, Black-Pond C (2007) Neurobiology and Neurodevelopmental Impact of Childhood Traumatic Stress and Prenatal Alcohol Exposure. Lang Speech Hear Serv Sch 38: 99-108. [Crossref]

27. Thiel K, Baladerian N, Boyce K, Cantos O, Davis L, et al. (2010) Fetal Alcohol Spectrum Disorders and victimization: Implications for families, educators, social services, law enforcement, and the judicial system. J Psychiatry Law 39: 121-157.

Copyright: (C2017 Brown J. This is an open-access article distributed under the terms of the Creative Commons Attribution License, which permits unrestricted use, distribution, and reproduction in any medium, provided the original author and source are credited. 